\title{
Search for the Production of a New Quark Flavor at the c.m.-System Energies between 33 and $35.8 \mathrm{GeV}$
}

D. P. Barber, U. Becker, (a) H. Benda, A. Böhm, J. G. Branson, J. Bron, D. Buikman, J. D. Burger, C. C. Chang, H. S. Chen, M. Chen, C. P. Cheng, Y. S. Chu, R. Clare, P. Duinker, G. Y. Fang, H. Fesefeldt, D. Fong, M. Fukushima, J. C. Guo, A. Hariri, G. Herten, M. C. Ho, H. K. Hsu, T. T. Hsu, R. W. Kadel, W. Krenz, J. Li, Q. Z. Li, M. Lu, D. Luckey, C. M. Ma, D. A. Ma, G. G. G. Massaro, T. Matsuda, H. Newman, M. Pohl, F. P. Poschmann, J. P. Revol, M. Rhode, H. Rykaczewski, K. Sinram, H. W. Tang, L. G. Tang, Samuel C. C. Ting, K. L. Tung, F. Vannucci, (a) X. R. Wang, P. S. Wei, M. White, G. H. Wu, T. W. Wu, J. P. Xi, P. C. Yang, C. C. Yu, X. H. Yu, N. L. Zhang, and R. Y. Zhu

III Physikalisches Institut Technische Hochschule, Aachen, West Germany, and Deutsches Elektronen-Synchrotron, Hamburg, West Germany, and Laboratory for Nuclear Science, Massachusetts Institute of Technology, Cambridge, Massachusetts, 02139, and Nationaal Instituut voor Kernfysica en Hoge-Energiefysica, Sectie H, Amsterdam, The Netherlands, and Institute of High Energy Physics, Chinese Academy of Science, Peking, People's Republic of China (Received 1 April 1980)

\begin{abstract}
This paper reports the results of a study of hadron production in $e^{+} e^{-}$collisions at c.m. system energies of 33,35 , and $35.8 \mathrm{GeV}$. Production of a new quark flavor has been sought. The measured values of the total cross section, the thrust distributions, and the study of inclusive muon production show no evidence for the production of a new charge$\frac{2}{3} e$ quark near threshold. In addition, during an energy scan in the region $29.9 \leqslant \sqrt{s} \leqslant 31.6$ $\mathrm{GeV}$, no hadron resonance indicating the existence of a bound state composed of charge$\frac{2}{3} e$ quarks has been found.
\end{abstract}

PACS numbers: $13.65+\mathrm{i}$

The MARK-J detector ${ }^{1}$ has taken data at energies of $\sqrt{s}=33,35$, and $35.8 \mathrm{GeV}$, the highest energies reached so far at PETRA, with time-integrated luminosities of 91,381 , and $133 \mathrm{nb}^{-1}$, respectively. The total cross section for the process

$$
e^{+} e^{-} \rightarrow \text { hadrons }
$$

via one-photon annihilation has been measured and the properties of the hadronic final states have been analyzed, to search for the production of a new quark flavor.

In addition to the detector setup reported earlier, ${ }^{1}$ an inner track detector composed of $992 \mathrm{drift}$ tubes has recently been installed. The tubes, which are arranged perpendicular to the beam line, make possible the reconstruction of the event vertex to an accuracy of two millimeters.

Events from reaction (1) are selected by requiring that (a) the total visible energy $E_{\text {vis }}$ is more than $50 \%$ of the available c.m.-system energy $\sqrt{s}$; (b) the energy is balanced within $50 \%$ of $E_{\text {vis }}$ in both the longitudinal and the transverse directions with respect to the beam line; and (c) the shape of the shower development in the layer structure of the detector is incompatible with a purely electromagnetic nature of the final state.

The acceptance for Reaction (1) has been calculated with use of a Monte Carlo simulation to pro- vide a phenomenological model of the hadron production process based on perturbative quantum chromodynamics (QCD). ${ }^{2}$ The model, which incorporates $q^{2}$ evolution and the weak decays of heavy quarks ${ }^{3}$ in the fragmentation process, also includes the effects of initial-state photon bremsstrahlung ${ }^{4}$ correct in quantum electrodynamics (QED) to order $\alpha^{3}$. After cuts the acceptance is $89 \%$ with little variation as a function of energy.

Background contributions to the hadron sample from the two-photon process

$$
e^{+} e^{-} \rightarrow e^{+} e^{-}+\text {hadrons }
$$

and

$$
e^{+} e^{-} \rightarrow \tau^{+} \tau^{-}
$$

have also been calculated and subtracted by Monte Carlo techniques.

The ratio $R$ of the total hadron production cross section to the pointlike cross section for $\mu$ pairs,

$$
R=\sigma\left(e^{+} e^{-} \rightarrow \text { hadrons }\right) / \sigma\left(e^{+} e^{-} \rightarrow \mu^{+} \mu^{-}\right),
$$

has been measured with use of a total sample of 28, 133, and 49 events, respectively, yielding

$$
\begin{aligned}
& R=2.9 \pm 0.6 \text { at } \sqrt{s}=33 \mathrm{GeV}, \\
& R=3.8 \pm 0.4 \text { at } \sqrt{s}=35 \mathrm{GeV}, \\
& R=4.4 \pm 0.7 \text { at } \sqrt{s}=35.8 \mathrm{GeV},
\end{aligned}
$$


once all corrections and background subtractions mentioned above have been applied. In addition to the statistical errors quoted above, we estimate a systematic error of $10 \%$ due to model dependence of the acceptance, event-selection criteria, and measurement of luminosity.

Fig. 1(a) summarizes the measurements of $R$ obtained by the MARK-J experiment at PETRA. They are compared with the predictions of QCD for the known five flavors, $u, d, s, c$, and $b$, as well as for production of a sixth flavor with a quark charge of $\frac{2}{3} e$ in the continuum. No increase in $R$ is seen corresponding to the opening of a new threshold up to the highest energy of $35.8 \mathrm{GeV}$. The results at $12 \mathrm{GeV} \leqslant \sqrt{s} \leqslant 31.6 \mathrm{GeV}$ agree with those reported by other PETRA experiments. ${ }^{5}$

In addition to this search for continuum production of a new quark flavor, an energy scan was performed between $\sqrt{s}=29.9$ and $31.6 \mathrm{GeV}$ measuring $R$ to search for bound states of a new quark lying below the continuum threshold. Data have been taken with about $25 \mathrm{nb}^{-1}$ average luminosity
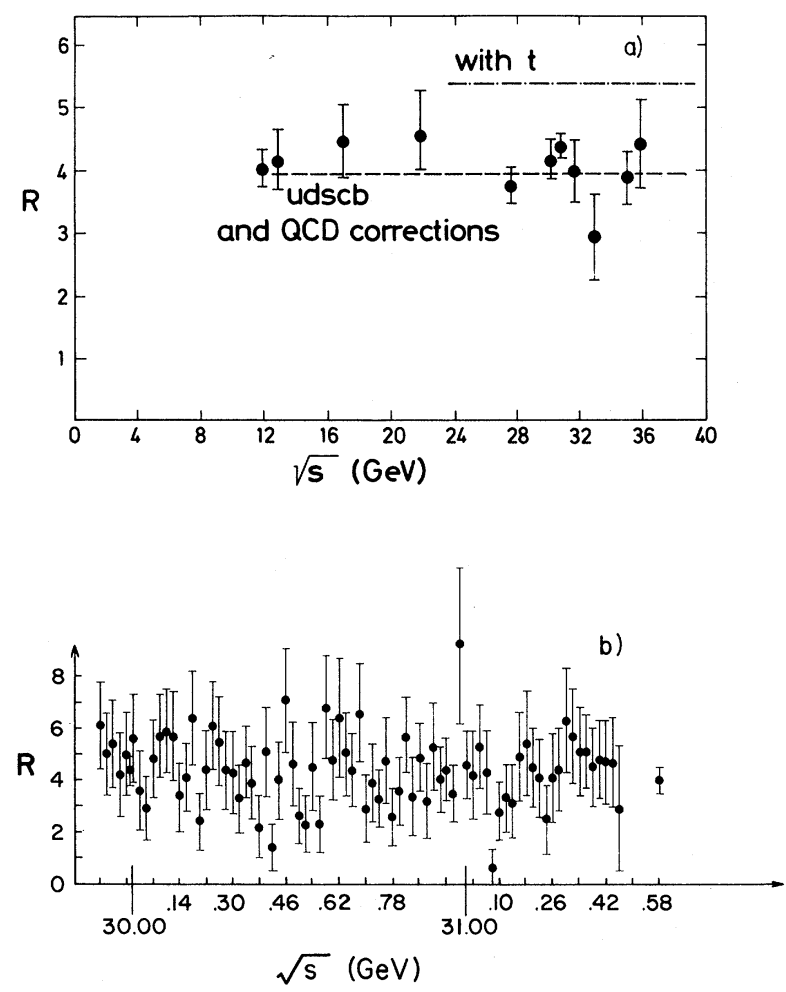

FIG. 1. (a) The relative hadronic cross section $R$ as a function of $\sqrt{s}$. The dashed line shows the QCD prediction without open top-quark production. The dotdashed line shows the prediction with new flavor production. (b) $R$ values measured during the energy scan between 29.9 and $31.6 \mathrm{GeV}$. per point in steps of $20 \mathrm{MeV}$ c.m.-system energy (matching the rms energy spread of PETRA). The overall hadron event sample for the scan consists of 807 events corresponding to a time-integrated luminosity of $1945 \mathrm{nb}^{-1}$. The results of the scan are shown in Fig. 1(b), along with the results obtained at 30.0 and $31.6 \mathrm{GeV}$. The figure shows that the data are consistent with the predictions of QCD for $u, d, s, c$, and $b$ quarks, i.e., with a constant value of $R$ over the whole range. No single point lies more than 3 standard deviations above the QCD line, while the value of $R$ averaged over the range of ths scan is $4.33 \pm 0.17$. In order to set a quantitative upper limit on the production of a narrow resonance, the data in Fig. 1(b) were fitted by a constant plus a Gaussian distribution:

$$
R=R_{0}+R_{v} \exp \left[-(w-M)^{2} / 2 \Delta_{w}{ }^{2}\right] \quad(w=\sqrt{s}),
$$

where $R_{0}$ represents the nonresonant continuum, $M$ is the mass of the resonance, $\Delta_{w}$ is the rms machine energy width, and $R_{v}$ is the peak value of the resonant contribution. The largest value of $\boldsymbol{R}_{v}$ consistent with the data was determined $k y$ trying fits with $M$, the center of the Gaussian fixed at all center-of-mass energies at which data were taken. The largest value of $\boldsymbol{R}_{v}$ was obtained at $31.32 \mathrm{GeV}$, corresponding to an upper limit on the resonance strength

$$
\Sigma_{V} \equiv \int\left(R-R_{0}\right) \sigma_{\mu \mu} d w
$$

of $33 \mathrm{MeV} \cdot \mathrm{nb}$ at $90 \%$ confidence level. Using the relation between the resonance strength, the width into $e^{+} e^{-}\left(\Gamma_{e e}\right)$, the hadronic width $\left(\Gamma_{h}\right)$, the total width $(\Gamma)$, and the hadronic branching ratio

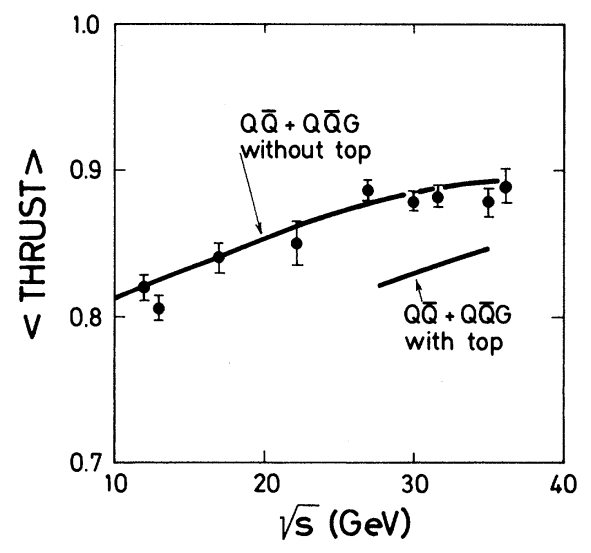

FIG. 2. Average value of thrust as a function of $\sqrt{s}$ together with the QCD prediction (solid line). The values expected from a $Q C D$ model with top quark are also shown. 


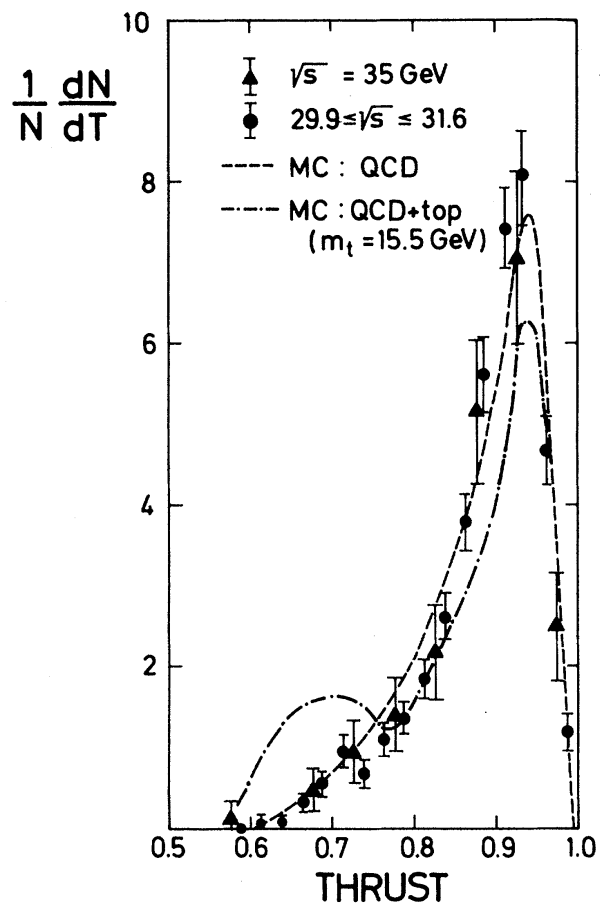

FIG. 3. The thrust distribution $N^{-1} d N / d T$ for $\sqrt{s}$ from 29.9 to $31.6 \mathrm{GeV}$ and for $\sqrt{s}=35 \mathrm{GeV}$. The curves are predictions for QCD models without top-quark (dashed line) and with top-quark (dot-dashed line) production.

$$
\begin{aligned}
& \left(B_{h} \equiv \Gamma_{h} / \Gamma\right), \\
& \quad \Sigma_{V} \equiv \int \frac{3 \pi}{M^{2}} \frac{\Gamma_{e e} \Gamma_{h}}{(w-M)^{2}+\Gamma^{2} / 4} d w=\frac{6 \pi^{2}}{M^{2}} B_{h} \Gamma_{e e},
\end{aligned}
$$

and taking radiative corrections into account we obtain

$$
B_{h} \Gamma_{e e}<1.3 \mathrm{keV} \text { ( } 90 \% \text { confidence limit). }
$$

This upper limit excludes the production of a vector particle consisting of a $q \bar{q}$ bound state where the quark has charge $\frac{2}{3} e$. On the basis of the experimental fact that $\Gamma_{e e} / e_{q}{ }^{2}$ is approximately constant for the vector-meson ground states $\rho, \omega$, $\varphi, J$, and $\Upsilon$ as predicted by duality arguments, one expects $B_{h} \Gamma_{e e} \simeq 4 \mathrm{keV}$ for the lowest-mass meson in the toponium family. Our results for the energy scan are in good agreement with those obtained from other experiments. ${ }^{7}$

A jet analysis of the hadronic events was performed with use of the spatial distribution of the energy deposited in the detector. The jetlike appearance of events is parametrized in terms of the parameter called thrust,

$$
T=\max \left[\sum_{i}\left|E_{\|^{i}}\right| / \sum_{i}\left|E^{i}\right|\right],
$$

where $E^{i}$ is an energy flow vector, whose direction is given by the position of a hit in a counter and magnitude by the corresponding deposited energy. $E_{\|}{ }^{i}$ is the parallel component of the energy flow vector along the axis which maximizes $T$ and the sums are taken over all counter hits. $\langle T\rangle$ is defined as the mean thrust of all events at a given center-of-mass energy.

The variation of $\langle T\rangle$ with energy is shown in Fig. 2. No step in $\langle T\rangle$, which is expected from the opening of a new threshold as indicated in the figure, is observed. The normalized thrust distribution $N^{-1} d N / d T$ for the data taken at $35 \mathrm{GeV}$ is shown in Fig. 3. It is compared with data taken earlier at $\sqrt{s}=30$ and $31.6 \mathrm{GeV}$ and during the energy scan. Within the limits of statistics, no change in the shape of the distribution is observed. Also indicated in the figures are predictions of the aforementioned QCD Monte Carlo program, ${ }^{2}$ which includes the effects of gluon bremsstrahlung as required by our data. ${ }^{8}$ The data agree well with the prediction for the five quark flavors $u, d, s, c$, and $b$. The discrepancy between the observed number of events with $T<0.80$ and the number expected from the production of a new quark of charge $\frac{2}{3} e$ makes the production of such a quark in the continuum unlikely up to an energy of $35.8 \mathrm{GeV}$ : At $35 \mathrm{GeV} 19$ events are observed while 16.1 are expected in the case of five quarks, and 37.0 in the case of six quarks. Similarly, at $\sqrt{s}=35.8 \mathrm{GeV}, 8$ events were observed while 6.2 are expected in the case of five quarks and 14.3 in the case of six quarks.

TABLE I. Percentages of hadron events with muons, compared to Monte Carlo estimates with and without the assumption to top-quark production.

\begin{tabular}{cccccc}
\hline \hline $\begin{array}{c}\sqrt{s} \\
(\mathrm{GeV})\end{array}$ & $\begin{array}{c}\text { Number of } \\
\text { hadron } \\
\text { events }\end{array}$ & $\begin{array}{c}\text { Number of } \\
\text { muon } \\
\text { events }\end{array}$ & $\begin{array}{c}\text { Percentage of } \\
\text { muon } \\
\text { events }\end{array}$ & $\begin{array}{c}\text { Monte Carlo } \\
\text { (no top) } \\
(\%)\end{array}$ & $\begin{array}{c}\text { Monte Carlo } \\
\text { (with top) } \\
(\%)\end{array}$ \\
\hline $\begin{array}{c}29.9-31.6 \\
35\end{array}$ & $\begin{array}{c}1147 \\
133\end{array}$ & $\begin{array}{c}43 \\
5\end{array}$ & $3.7 \pm 0.6$ & $5.0 \pm 0.4$ & $7.7 \pm 0.5$ \\
\hline \hline
\end{tabular}


Corroborating evidence comes from the study of inclusive muons in the hadron sample. Table I shows the percentage of hadronic events accompanied by a muon. The observed percentages are in good agreement with the rate of production expected from decays of mesons composed of $u, d$, $s, c$, and $b$ quarks and antiquarks obtained from the above-mentioned Monte Carlo program which includes the effects of pion and kaon decay in flight and hadron punchthrough. The existence of a new quark with charge $\frac{2}{3} e$ would enhance the number of inclusive muons in the scheme of cascading weak quark decays. ${ }^{3}$ As indicated in Table I, this assumption is disfavored by the data.

In conclusion, all data up to a c.m.-system energy of $35.8 \mathrm{GeV}$ agree well with the predictions of QCD for the production of the five known quark flavors. The measurement of total cross sections, thrust distributions, and inclusive muon production indicate that the continuum production of a new charge- $-\frac{2}{3} e$ quark is unlikely up to this energy. The $R$ values obtained during the energy scan show that no narrow resonance with the width expected from a charge $-\frac{2}{3} e$ bound state produced between 29.9 and $31.6 \mathrm{GeV}$. The production of a charge- $-\frac{1}{3} e$ quark can, however, not be ruled out by the present data.

We wish to thank Professor H. Schopper, Professor G. Voss, Professor A. N. Diddens, Professor H. Faissner, Professor E. Lohrmann, Professor F. Low, Dr. F. J. Eppling, and Dr. G. Soehngen for their valuable support and Dr. A.
Ali, Professor A. De Rújula, Professor S. Glashow, Dr. T. Walsh, and Dr. L.-L. Chau Wang for helpful discussions.

(a) Present address: CERN, Geneva, Switzerland. ${ }^{1}$ D. P. Barber et al., Phys. Rev. Lett. 42, 1110, 1113 (1979), and Massachusetts Institute of Technology Laboratory for Nuclear Science Report No. 107, 1980 (unpublished).

${ }^{2}$ A. Ali, E. Pietarinen, G. Kramer, and J. Willrodt, DESY Report No. 79/86, 1979 (unpublished).

${ }^{3}$ A. Ali et al ., DESY Report No. 79/63, 1979 (unpublished); M. Kobayashi and T. Maskawa, Prog. Theor. Phys. 49,652 (1973).

${ }^{4}$ The accurate generation of radiative events is made possible through the recent unpublished work of F. A. Berends and R. Kleiss. Also see F. A. Berends, K. J. F. Gaemers, and R. Gastmans, Nucl. Phys. B57, 381 (1973), and B63, 381 (1973).

${ }^{5} \mathrm{~W}$. Bartel et al., Phys. Lett. 88B, 171 (1979), and

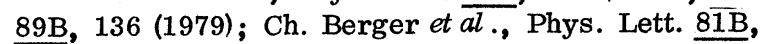
$\overline{410}$ (1979), and 86B, 413 (1979), and 86B, 418 (1979); R. Brandelik et al., Phys. Lett. 83B, 261 (1979), and 86B, 243 (1979), and 89B, 418 (1980).

${ }^{6} \mathrm{~J}$. L. Rosner, C. Quigg, and H. B. Thacker, Phys. Lett. 74B, 350 (1978); C. Quigg, in Proceedings of the International Symposium on Lepton and Photon Interactions, Fermilab, 1979 (to be published); M. Greco, Phys. Lett. 77B, 84 (1978).

${ }^{7}$ Ch. Berger et al ., Phys. Lett. 91B, 148 (1980); W. Bartel et al ., Phys. Lett. 91B, 152 (1980); R. Brandelik et al., Phys. Lett. 88B, 199 (1979).

${ }^{8}$ D. P. Barber et al., Phys. Rev. Lett. $\underline{43}, 830$ (1979), and Phys. Lett. 89B, 139 (1979). 